\title{
Simulation Study to Measure Parametrical Effect on the Characteristics of MHD Flow
}

\author{
Binyam Zigta and Purnachandra Rao Koya \\ School of Mathematical and Statistical Sciences, Hawassa University, ETHIOPIA
}

\begin{abstract}
This paper investigates Magneto hydrodynamics of fluid flow of unsteady free convection between a pair of infinite vertical Couette plates embedded in a porous medium. Temperatures of the plates and concentration of the fluid vary with time. The free convection between plates with the inclusion of thermal radiation and chemical reaction is considered. The Couette plates are immersed vertically in a porous medium. We use regular perturbation technique to convert the governing nonlinear partial differential equations into a system of ordinary differential equations. The effects of various parameters viz. permeability of porous medium, thermal Grashof number, frequency parameter, radiation parameter, chemical reaction parameter and Schmidt number on velocity, temperature, concentration and skin friction of the fluid flow have been discussed. Simulation study is conducted using Mat lab coding in support of the theoretical expectations. The results show that an increase in permeability of porous medium, thermal Grashof number and frequency parameter effects in decrease in velocity. Furthermore, as the thermal radiation increases the temperature decreases near the moving plate while it increases in the region closer to the boundary layer of the stationary plate. Modified Grashof number computed based on concentration difference increases as the concentration of the fluid flow decreases. The concentration of the fluid decreases with an increase in Schmidt number. Physically, this implies an increase in Schmidt number results in decreasing of molecular diffusivity and is also results in decreasing of concentration.
\end{abstract}

Keywords: MHD, Permeability of porous medium, thermal radiation, frequency parameter and Chemical reaction parameter.

\section{Introduction.}

Flows of fluids through a porous medium are of main and principal interest because they occur frequently in nature. Such fluid flows have attracted the attention of many researchers due to their application in Science and Technology: Ground water flow, Plasma studies, Geophysics, Geology, Aerodynamics, Geothermal reservoirs, Thermal engineering, Petroleum engineering and Agricultural engineering.

Many scholars have studied MHD free convective energy and concentration flow in a porous medium to examine flow of oil, water and natural gas. The study of free convective energy flow through a porous plate, in relation to the application of the areas mentioned, has been made [1]. Unsteady free convective concentration fluid flow past a pair of vertical and parallel plates is studied [2]. The unsteady free convection flow through a porous medium has been considered and investigated [3]. The unsteady free convection flow through a highly porous medium bounded by an infinite porous plate is investigated [4]. Free convective flow through a porous medium between two vertical parallel plates is studied [5].

The effect of unsteady free convective MHD and concentration fluid flow through porous medium with constant suction and constant heat flux in rotating system is studied [6].Unsteady free convective MHD fluid flow past a vertical porous plate has been investigated [7]. The effects of Radiation on free convection over vertical flat plates embedded in porous medium with high porosity are studied [8]. The effect of radiation on chemically reacting MHD boundary layer flow of energy and concentration through a porous vertical flat plate is investigated [9].The effect of radiation on natural convection near a vertical plate embedded in porous medium with wall temperature moving violently has been studied [10].

The effects of radiation on unsteady MHD free convective flow past an oscillating vertical porous plate embedded in a porous medium with oscillatory heat flux are investigated [11]. Radiation effects on concentration fluid flow through a highly porous medium with heat generation and chemical reaction have been studied [12].The effect of chemical reaction on the fluid flow in the presence of heat transfer and magnetic field has been investigated [13].The effects of thermal radiation and magnetic field on unsteady boundary layer mixed convection flow and heat transfer problem from a vertical porous stretching surface are studied [14]. The numerical solution of the steady mixed convection boundary layer flow over a vertical impermeable surface embedded in a porous medium when viscosity of a fluid varies inversely as a linear function of temperature are studied [15].

The simultaneous effects of opposing buoyancy force on heat and mass transfer by free convection in a fluid saturated by porous medium are studied [16]. The effect of transpiration velocity on the energy and 
concentration species characteristics of mixed convection numerically analyzed on a permeable vertical plate embedded in a saturated porous medium under the influence of both thermal and concentration diffusion studied [17]. The effects of chemical reaction on an unsteady MHD free convection flow past an infinite vertical porous plate with constant suction are studied [18]. The effects of chemical reaction on an unsteady MHD flow past a semi-infinite vertical porous plate with viscous dissipation has been analyzed numerically [19]. The effect of radiation on unsteady MHD natural convective flow on a vertical porous plate embedded in a porous medium is studied [20].

From literature reviews it can be observed that in a very few papers the free convective vertical Couette plates embedded in a porous medium has been studied. To fill the gap in our present study we consider this as our subject.

In this study, the effect of magnetic field on the flow of fluid between two vertical parallel Couette plates will be considered and studied. The fluid considered here has the properties viz., viscous, incompressible, electrically conducting, Newtonian, chemical reacting and thermal radiating. Free stream velocity of the fluid is assumed to be fluctuating. Furthermore, we assume temperature and concentration of the fluid are also fluctuating with time. Although, the effects of thermal radiation, chemical reaction parameter and permeability of porous medium are very important and significant, they have not been considered. We have been initiated to study the effects of thermal radiation, chemical reaction parameter and permeability of porous medium [22].

Permeability in fluid mechanics is commonly symbolized by $k$ and is a measure to allow fluids to pass through a porous medium. The concept of permeability is important in determining the flow characteristics of hydrocarbons in oil and gas reservoirs and of ground water flow. Permeability also varies with stress and temperature but does not depend on the fluid. In fluid Mechanics fluid flow through a porous medium is the manner in which fluids behave when flowing through a porous medium: for example, sponge or sand used to filter water or another porous material.

The main objective of this paper is to study the effect of MHD on free convection oscillatory Couette flow immersed in a porous medium when the temperature and concentration oscillates with time, in presence of the thermal radiation, chemical reaction and permeability of porous medium.

\section{Mathematical Formulation and Analysis of the Model}

Consider two dimensional free convection Couette flows of unsteady, viscous, incompressible, electrical conducting, Newtonian, chemical reacting and radiating fluid bounded by two infinite vertical plates separated by a distance $b$ immersed in a porous medium. The pictorial representation of the model and coordinate system are shown in Figure 1. The $\bar{x}$ axis is taken along the plate in vertical or upward direction that is opposite to the direction of gravity. The $\bar{y}$ axis is taken normal to the vertical plate.

The vertical moving plate is located at $\bar{y}=0$ along $\bar{x}$ axis where the temperature is given by $\bar{T}_{m}$ and the concentration is also given by $\bar{C}_{m}$. The other stationary plate is located at $\bar{y}=b$ where the temperature is denoted by $\bar{T}_{b}$ and the concentration is denoted by $\bar{C}_{b}$.

Naturally, it may be observed that temperature and time are directly proportional i.e. an increase in temperature results in an increase in time in the presence of thermal radiation as well as pure convection of the whole fluid flow. The temperature changes cause density variation which leads to buoyancy forces acting on fluid elements. Initially at time $\bar{t}=0$, the plate and the fluid are at the same temperature $\bar{T}_{b}$ in stationary position and with concentration level $\bar{C}_{b}$ at all points. At time $\bar{t}>0$, the plate temperature and concentration are raised $\bar{T}_{m}$ and $\bar{C}_{m}$ respectively and are maintained at a constant value. This result is due to porous medium i.e., porous medium is used to insulate a heated body by maintaining its temperature. As time changes temperature changes this leads to buoyancy force acting in fluid flow so the plate $\bar{y}=0$ starts moving in its own plane along positive $\bar{x}$ direction with velocity $V$. And for all the later time $\bar{t}>0$ the plate continues to be in motion. But due to viscosity of the fluid and acceleration due to gravity the motion will decrease and consequently it returns after some time $\bar{t}$.

We now set the boundary conditions for velocity, temperature and concentration of the fluid in the model. At the initial time $\bar{t}=0$ the conditions are set to be

$\bar{u}=0, \bar{v}=0, \bar{T}=\bar{T}_{b}, \bar{C}=\bar{C}_{b}$ for all $\bar{y}$

Similarly, at any latter time $\bar{t}>0$ the conditions are

$\left\{\bar{u}=V, \bar{T}=\bar{T}_{m}, \bar{C}=\bar{C}_{m}\right.$ at $\bar{y}=0$

$\left\{\bar{u} \rightarrow 1, \bar{T}=\bar{T}_{b}, \bar{C}=\bar{C}_{b}\right.$ as $\bar{y} \rightarrow 1$

The moving plate is being heated by supplying of heat so as to maintain its temperature constant, while the stationary plate $\bar{y}=b$ is maintained at the constant temperature $\bar{T}_{b}$.

It is assumed that the radiation heat flux assumed to be present in the form of unidirectional flux in $\bar{y}$ direction.We assume some list of models which helps us to derive the governing equations viz. the fluid properties such as temperature, viscosity, pressure, specific volume, specific gravity are constant, the influence 
of the density variation with both temperature and concentration in the body force term or Boussinesq's approximation is a variable but not a constant. the flow of the fluid is unsteady, laminar, incompressible, viscous, electrically conducting, Newtonian, chemical reacting and thermal radiating, the magnetic field applied is transversal between the vertical plates, the induced magnetic field of the fluid is negligible and hence the magnetic Reynolds number is a very small constant, the external electric field is supposed to be zero and the electric field due to polarization of charges is negligible. Viscosity is also considered with the constant permeability of porous medium. We assume that there exist a homogeneous chemical reaction of first order with constant rate between diffusing concentration or species and the fluid in the moving plate. A reaction is said to be a first order if the rate of reaction is directly proportional to concentration.

Let us consider that the fluid being gray in color has radiation absorbing nature, emits radiation, and is a non-scattering medium. Roseland approximation is used to describe the radiative heat flux in the energy equation.

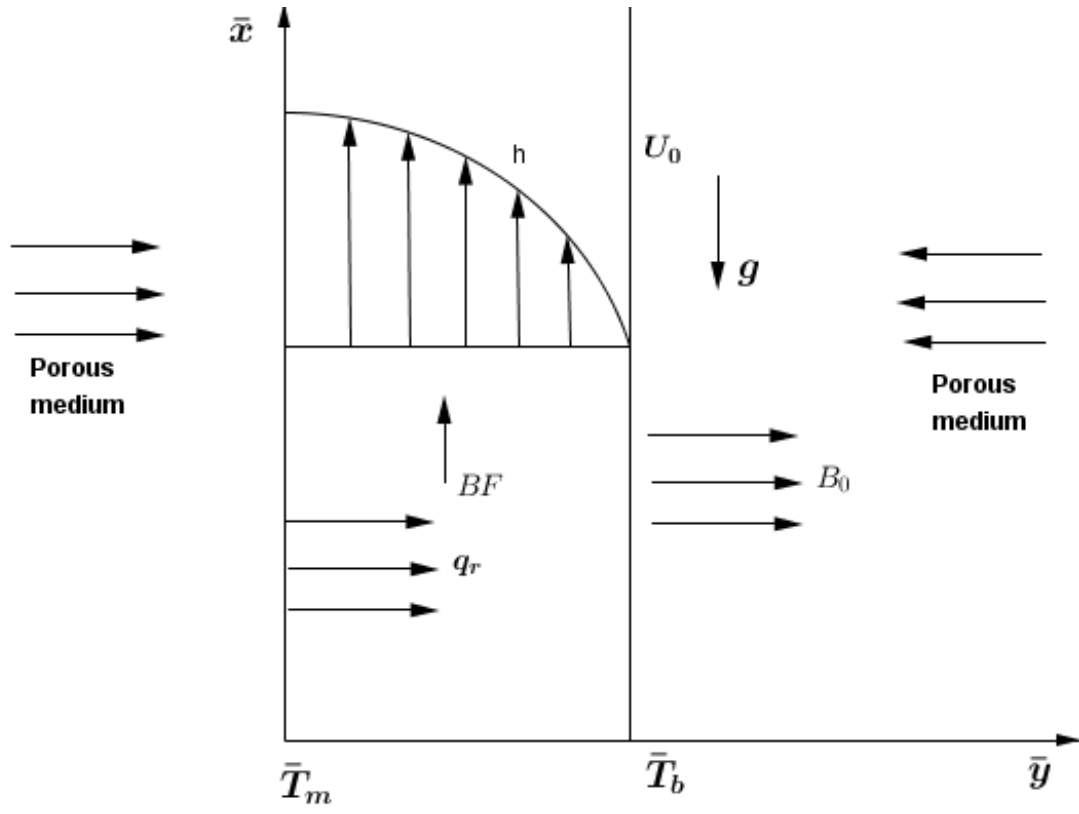

Figure 1: Pictorial representation of the model with coordinate system

We assume that the free stream velocity follows according to the expression

$\bar{U}(\bar{t})=U_{o}\left(1+\varepsilon e^{i \bar{\omega} \bar{t}}\right)$

Here in (3), $U_{o}$ represents the mean constant free stream velocity, $\bar{\omega}$ is the frequency and $\bar{t}$ is the time. We now derive the governing equations of the present model based on the preceding assumptions. The momentum equation of the model takes the form as

$\frac{\partial \bar{u}}{\partial \bar{t}}=\frac{\partial \bar{U}}{\partial \bar{t}}+v \frac{\partial^{2} \bar{u}}{\partial \bar{y}^{2}}+g \beta\left(\bar{T}-\bar{T}_{b}\right)+g \beta_{c}\left(\bar{C}-\bar{C}_{b}\right)-\frac{J X B}{\rho}-\frac{v}{\bar{k}} \bar{u}$

In (4), the vector cross product $J x B$ represents the Lorentz force. This term is a body force corresponding to magneto hydrodynamic fluid flow. The total magnetic field is represented by $B$. The density of the current is represented by $J$ and $\vec{k}$ denotes the permeability of porous medium.

$J x B=-\sigma B^{2} u$

Also, the expression for Lorentz force reduces and takes the form as

In view of the result (5), the equation (4) reduces to

$\frac{\partial \bar{u}}{\partial \bar{t}}=\frac{\partial \bar{U}}{\partial \bar{t}}+v \frac{\partial^{2} \bar{u}}{\partial \bar{y}^{2}}+g \beta\left(\bar{T}-\bar{T}_{b}\right)+g \beta_{c}\left(\bar{C}-\bar{C}_{b}\right)-\frac{\sigma B^{2} \bar{u}}{\rho}-\frac{v}{\bar{k}} \bar{u}$

The energy equation of the model can be expressed as

$\frac{\partial \bar{T}}{\partial \bar{t}}=\alpha \frac{\partial^{2} \bar{T}}{\partial \bar{y}^{2}}-\frac{1}{\rho C_{p}} \frac{\partial q_{r}}{\partial y}$

The concentration equation of the model can be expressed as

$\frac{\partial \bar{C}}{\partial \bar{t}}=D \frac{\partial^{2} \bar{C}}{\partial \bar{y}^{2}}-k_{r}\left(\bar{C}-\bar{C}_{b}\right)$

The equations (6) - (8) govern the present model described in Figure 1. Here in (6) - (8) we have used some parameters which can be assigned physical interpretations as follows: $\alpha$ is thermal diffusivity; $\rho$ is density of the fluid; $g$ is the acceleration due to gravity; $\beta$ is the thermal expansion coefficient; $\beta_{c}$ the concentration expansion coefficient; $\bar{T}$ is the temperature of the fluid in the boundary layer; $\bar{T}_{m}$ is the 
temperature of the moving plate; $\bar{T}_{b}$ is the temperature of the stationary plate; $q_{r}$ is the radiative heat flux; $D$ is the mass diffusivity; $\bar{k}$ is the permeability of porous medium. The term $\left[\left(1 / \rho C_{p}\right)\left(\partial q_{r} / \partial y\right)\right]$ is the thermal radiation effect; and $k_{r}\left(\bar{C}-\bar{C}_{b}\right)$ is the generative chemical reaction.

The physical interpretation of the minus sign in (6) shows that the fluid flows in the direction from higher to lower potential. In (7), the minus sign of the radiative heat flux term $\left[\left(1 / \rho C_{p}\right)\left(\partial q_{r} / \partial y\right)\right]$ indicates the emission of energy away from the objects that is heat is lost. Furthermore, in the energy equation (7), $\alpha>0$ shows heat generation and $\alpha<0$ indicates heat absorption i.e., thermal energy diffuses more rapidly through substances with high thermal diffusivity $\alpha$ and it diffuses slowly through those with low thermal diffusivity $\alpha$.

In (8), the minus sign indicates that the mass diffusion represents 'Generative chemical reaction'. Here chemical reaction is an exothermic reaction and in that case heat is generated. However, the chemical reaction is said to be generative or exothermic if the chemical reaction parameter is negative i.e. $k_{r}<0$.If $k_{r}>0$ the chemical reaction is said to be destructive or endothermic chemical reaction

The boundary conditions for the model (6) - (8), can be chosen based on the Figure 1, as

$\bar{y}=0, \bar{u}=U_{0}\left(1+\varepsilon e^{i \overline{\bar{\omega}} \bar{t}}\right)$

$\bar{T}=\bar{T}_{m}+\varepsilon\left(\bar{T}_{m}-\bar{T}_{b}\right) e^{i \overline{\bar{\omega}} \bar{t}}$

$\bar{C}=\bar{C}_{m}+\varepsilon\left(\bar{C}_{m}-\bar{C}_{b}\right) e^{i \overline{\bar{\omega}} \bar{t}}$

$\bar{y}=b, \quad \bar{u}=0, \bar{T}=\bar{T}_{b}, \quad \bar{c}=\bar{c}_{b}$

Using the Roseland approximation for radiative heat transfer and the Roseland approximation for diffusion and also following the other scholarly works [21], the expression for radiative heat flux $q_{r}$ can be given as

$q_{r}=\left(\frac{-4 \sigma}{3 k_{s}}\right)\left(\frac{\partial \bar{T}^{4}}{\partial \bar{y}}\right)$

Here in (13), the parameters $\sigma$ and $k_{s}$ represent the Stefan Boltzmann constant and the Roseland mean absorption coefficient respectively.

We now assume that the temperature differences within the fluid flow are sufficiently small so that $\bar{T}^{4}$ in (13) can be expressed as a linear function of $\bar{T}_{b}$ using Taylor series expansion. The Taylor series expansion of $\bar{T}^{4}$ about $\bar{T}_{b}$, after neglecting the higher order terms, takes the form as

$\bar{T}^{4} \cong 4 \bar{T}_{b}^{3} \bar{T}-3 \bar{T}_{b}^{4}$

Using (13) and (14) in (7), we obtain

$\frac{\partial \bar{T}}{\partial \bar{t}}=\alpha\left(\frac{\partial^{2} \bar{T}}{\partial \bar{y}^{2}}\right)+\left(\frac{1}{\rho C_{p}}\right)\left(\frac{16 \sigma \bar{T}_{b}{ }^{3}}{3 k_{s}}\right)\left(\frac{\partial^{2} \bar{T}}{\partial \bar{y}^{2}}\right)+\left(\frac{Q_{0}}{\rho C_{p}}\right)\left(\bar{T}-\bar{T}_{b}\right)$

Here in (13), the term $\left[\left(Q_{0} / \rho C_{p}\right)\left(\bar{T}-\bar{T}_{b}\right)\right]$ denotes heat absorption of the fluid.

\section{Non Dimensionalization of the Model}

In order to solve the governing equations (6) - (8) of the model it is convenient and easy to deal with its dimensionless form. Hence, we find the dimensionless form of the model by introducing the following nondimensional quantities

$$
\begin{gathered}
y=\bar{y} / b, \quad R=4 \sigma T_{b}{ }^{3} / \kappa k_{s}, \quad u=\bar{u} / U_{0}, \quad U=\bar{U} / U_{0}, \quad t=\bar{\omega} \bar{t}, \quad \varpi=\bar{\omega} b^{2} / v, \\
G c=\left\{\left[g \beta_{c}\left(\bar{C}_{m}-\bar{C}_{b}\right)\right] / v U_{0}\right\}, G r=\left\{g \beta b^{2}\left(\bar{T}_{m}-\bar{T}_{b}\right) / v U_{0}\right\}, \quad S c=v / D, \quad P r=v / \alpha, \\
M=\sqrt{\left(\sigma B^{2} b^{2} / \rho v\right)}, C=\left[\left(\bar{C}-\bar{C}_{b}\right) /\left(\bar{C}_{m}-\bar{C}_{b}\right)\right], K=\bar{k} U_{0}{ }^{2} / v^{2}, \theta=\left[\left(\bar{T}-\bar{T}_{b}\right) /\left(\bar{T}_{m}-\bar{T}_{b}\right)\right]
\end{gathered}
$$

After substituting the above non-dimensional quantities in (6) - (8) and after simple algebraic manipulations, the non-dimensional form of the model takes the following form as

$$
\begin{aligned}
& \omega \frac{\partial u}{\partial t}=\omega \frac{\partial U}{\partial t}+\frac{\partial^{2} u}{\partial y^{2}}+G r \theta+G c C-\frac{u b^{2}}{v U_{0}}\left(\frac{\sigma b^{2}}{\rho}+\frac{U_{0}^{3}}{K}\right) \\
& \frac{\partial \theta}{\partial t}=\frac{\kappa}{\rho C_{p}} \frac{\partial^{2} \theta}{\partial y^{2}}+\frac{4 R \kappa}{3} \frac{\partial^{2} \theta}{\partial y^{2}}+\frac{Q_{0}}{\rho C_{p}} \\
& \omega S c \frac{\partial C}{\partial t}+k_{r} C b^{2} \frac{S c}{v}=\frac{g \beta_{c} b^{2}}{G c v U_{0}} \frac{\partial^{2} C}{\partial y^{2}}
\end{aligned}
$$

The corresponding boundary conditions (9) - (12) take the form as

$y=0, u=1+\varepsilon e^{i t}, \theta=1+\varepsilon e^{i t}, C=1+\varepsilon e^{i t}$

$y=1, u=0, \theta=0, C=0$

The system of equations (16) - (18) together with the boundary conditions (19) - (20) constitutes the non-dimensional form of the present model.

\section{Analytical Solution of the problem}

Here we now obtain analytical solution to the non-dimensional form of the present model (16) - (20). For that purpose let us assume that the amplitudes of the free stream velocity, temperature and concentration 
variation are very small positive quantities. Thus, the amplitude of free stream velocity needs to be considered to be $0<\varepsilon \ll 1$

Using perturbation techniques the solution of the model has the following form

$u(y, t)=u_{0}(y)+\varepsilon u_{1}(y) e^{i t}+o\left(\varepsilon^{2}\right)$

$\theta(y, t)=\theta_{0}(y)+\varepsilon \theta_{1}(y) e^{i t}+o\left(\varepsilon^{2}\right)$

$C(y, t)=C_{0}(y)+\varepsilon C_{1}(y) e^{i t}+o\left(\varepsilon^{2}\right)$

$U=1+\varepsilon e^{i t}$

Also, the free stream velocity takes the form as

On substituting equations (21) - (24) into (16) - (20), and equating harmonic and non-harmonic terms and neglecting higher orders of $\varepsilon$ we obtain the following system of equations:

$u_{0}^{\prime \prime}-u_{0} \frac{b^{2}}{v u_{0}}\left(\frac{\sigma B^{2}}{\rho}+\frac{U_{0}^{3}}{k}\right)=-G r \theta_{0}-G c C_{0}$

$u_{1}^{\prime \prime}-u_{1}\left(i \omega+\frac{b^{2}}{v u_{0}}\left(\frac{\sigma B^{2}}{\rho}+\frac{U_{0}^{3}}{k}\right)\right)=i \omega-G r \theta_{1}-G c C_{1}$

$C_{0} "-T C_{0}=0$

$C_{1}{ }^{\prime \prime}-P C_{1}=0$

$\theta_{0} "\left(\frac{\kappa}{\rho C_{p}}+\frac{4 R k}{3}\right)+\frac{Q_{0}}{\rho C_{p}}=0$

$\theta_{1}^{\prime \prime}\left(\frac{4 R k}{3}+1\right)-i \theta_{1}=0$

Further, the new boundary conditions corresponding to (19) - (20) obtained after Non dimensionalization as

$y=0, u_{0}=1, u_{1}=1, \theta_{1}=1, C_{0}=1, C_{1}=1$

$y=1, u_{0}=0, u_{1}=0, \theta_{0}=0, \theta_{1}=0, C_{0}=0, C_{1}=0$

Up on solving equations (25) - (30) together with the boundary conditions $(31)-(32)$, we obtain the required analytical solutions as follows:

$u_{0}(y)=A e^{\alpha_{1} y}+B e^{-\alpha_{1} y}-G r \theta_{0}-G c C_{0}$

$u_{1}(y)=A^{\prime} e^{\gamma_{1} y}+B^{\prime} e^{-\gamma_{1} y}-i \omega-G r \theta_{1}-G c C_{1}$

$\theta_{0}(y)=y^{2}\left(-C^{*}-1\right)+C^{*} y+1$

$\theta_{1}(y)=C_{1} e^{\beta x}+C_{2} e^{-\beta x}$

$C_{0}(y)=d_{1} e^{\lambda_{1} y}+d_{2} e^{-\lambda_{1} y}$

$C_{1}(y)=f_{1} e^{\lambda_{2} y}+f_{2} e^{-\lambda_{2} y}$

Here in (33) - (38), various symbols represent the following expressions:

$A=\frac{\left(G r \theta_{0}+G c C_{0}\right)\left(1-e^{-\alpha_{1}}\right)-e^{-\alpha_{1}}}{e^{\alpha_{1} 1-e^{-\alpha_{1}}}}, \quad B=\frac{\left(\operatorname{Gr} \theta_{0}+G c C_{0}\right)\left(1-e^{\alpha_{1}}\right)-e^{\alpha_{1}}}{e^{-\alpha_{1}-e^{\alpha_{1}}}}, \quad A^{*}=-\left[C^{\prime}+D^{\prime}+E^{\prime}+F^{\prime}\right]$,

$B^{*}=-\left[1+C^{\prime} e^{q^{\prime}}+D^{\prime} e^{-q^{\prime}}+E^{\prime} e^{r^{\prime}}+F^{\prime} e^{-r^{\prime}}\right], \quad A^{\prime}=\frac{C^{\prime}-A^{*}}{e^{p^{\prime}}-e^{-p^{\prime}}}, \quad B^{\prime}=\frac{-C_{1}+A^{*}}{e^{p_{1}^{\prime}}-e^{-p_{1}^{\prime}}}$

$\beta=\sqrt{\frac{3 i}{4 R k+1}}, \quad C_{1}=\frac{1}{e^{2 \beta}-1}, \quad C_{2}=\frac{e^{2 \beta}}{e^{2 \beta}-1}, \quad T=\frac{S c k_{r} G c U_{0}}{g \beta_{c}}, \quad \lambda_{1}= \pm \sqrt{\frac{S c k_{r} G c U_{0}}{g \beta_{c}}}$

$d_{1}=\frac{1}{1-e^{2 \lambda_{1}}}, \quad d_{2}=\frac{e^{2 \lambda_{1}}}{e^{2 \lambda_{1}}-1}, \quad \lambda_{2}= \pm \sqrt{\frac{S c\left(i \omega \nu+k_{r} b^{2}\right) G c U_{0}}{g \beta_{c} b^{2}}}, \quad f_{1}=\frac{1}{1-e^{2 \lambda_{2}}}$

$f_{2}=\frac{e^{2 \lambda_{2}}}{e^{2 \lambda_{2}}-1}, \quad P=\frac{S c\left(i \omega \nu+k_{r} b^{2}\right) G c U_{0}}{g \beta_{c} b^{2}}, \quad C^{*}=-1-\frac{R}{2}, P^{\prime}=\sqrt{i \omega+M^{2}}, \quad q^{\prime}=\sqrt{i \omega p r}$

$r^{\prime}=\sqrt{i \omega S c}, \quad C^{\prime}=\frac{G r f_{1}}{q^{\prime 2}-\left(i \omega+M^{2}\right)^{\prime}}, \quad Q_{o}=\frac{16 \sigma \bar{T}_{b}^{2}}{k_{s}}, \quad D^{\prime}=\frac{-G r}{\left(1-e^{-2 r}\right)\left(r^{\prime 2}-p^{\prime 2}\right)}, E^{\prime}=\frac{-G c}{\left(1-e^{2 q^{\prime}}\right)\left(q^{\prime 2}-p^{\prime 2}\right)}$,

$F^{\prime}=\frac{-G c}{\left(1-e^{-2 q^{\prime}}\right)\left(q^{\prime 2}-p^{2}\right)}, \quad \alpha_{1}=\sqrt{\frac{b^{2}}{v U_{0}}\left(\frac{\sigma B^{2}}{\rho}+\frac{U_{0}^{3}}{k}\right)}, \quad \gamma_{1}=\sqrt{i \omega+\frac{b^{2}}{v U_{0}}\left(\frac{\sigma B^{2}}{\rho}+\frac{U_{0}^{3}}{k}\right)}$

\section{Simulation study}

Here we consider an unsteady magneto hydrodynamics free convective fluid flow bounded between two infinite vertical Couette plates embedded in a porous medium. The effects of different parameters such as permeability of porous medium, thermal Grashof number, frequency parameter, radiation parameter, chemical reaction parameter and Schmidt number on velocity, temperature ,concentration and skin friction of the model have been studied using simulation. 


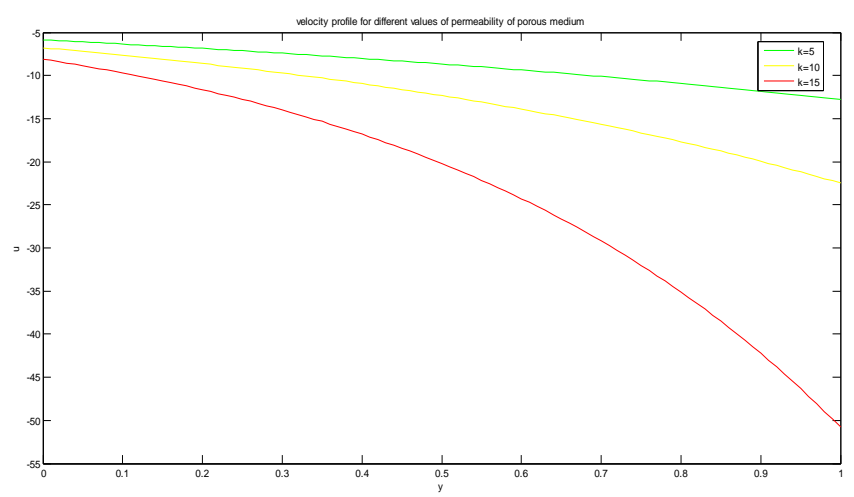

Figure 2 Velocity profile of the model for different values of permeability porous medium $k$

In Figure 2, we have presented the simulated results of the influence of permeability parameter on velocity. We draw the graph $y$ the distance between the plates against velocity $u$ holding other parameters constant. From the figure we observed that the maximum velocity $u$ is attained near the moving plate and then it decreases as it goes to the stationary plate. Furthermore, we observed that the velocity decreases with increasing values of permeability of porous medium. Physically, this is true because that the permeability of porous medium has a retarding property or resistance on the fluid flow. This result is due to heating of the stationary plate but the reverse effect is noticed in case of cooling of the moving plate.

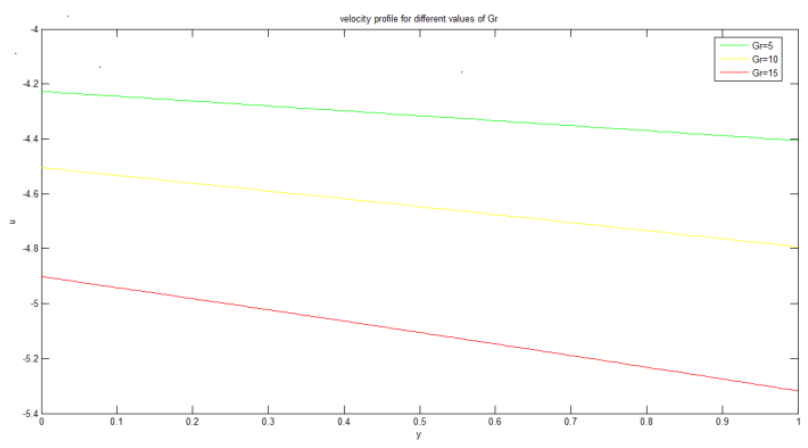

Figure 3 Velocity profile of the model for different values of thermal Grashof number $\mathrm{Gr}$

In Figure 3, the influence of thermal Grashof number $\mathrm{Gr}$ on velocity has been studied by simulation. We draw the graph $y$ the distance between the plates against velocity $u$ holding other parameters constant and we have observed $u$ is maximum in the moving plate then it decreases as it goes to the stationary plate. Although from reality we know that as thermal Grashof number increases the velocity of fluid flow increases. However, from simulation results we have observed the reverse effect that is as thermal Grashof number increases the velocity decreases. This is due to heating of the stationary plate and the presence of porous medium which results in increasing the resistance of the fluid flow. And hence decreases the fluid flow of the velocity.

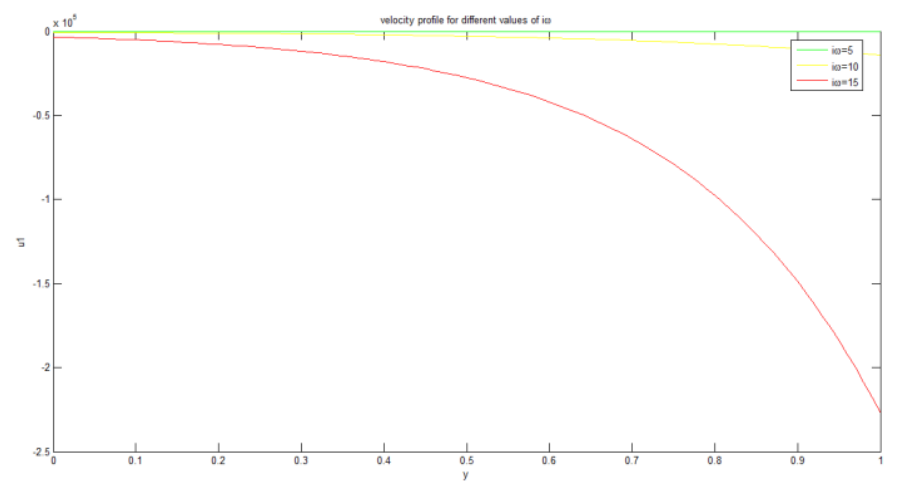


Figure 4 Velocity profile of the model for different values of frequency parameter $\omega$

In Figure 4, the influence of frequency parameter on velocity has been investigated by simulation. We draw the graphy the distance between the plates against velocity $u_{1}$ holding other parameters constant and we have seen $u_{1}$ is maximum in the moving plate and it decreases near the stationary plate. From the simulation results we have observed for small values of frequency parameter there is a little bit decrease of velocity as the distance between the plates increase but for larger values of frequency parameter the velocity decreases as the distance between the plates increase. In general, we have observed from the simulation results that as the frequency parameter increases the velocity decreases.

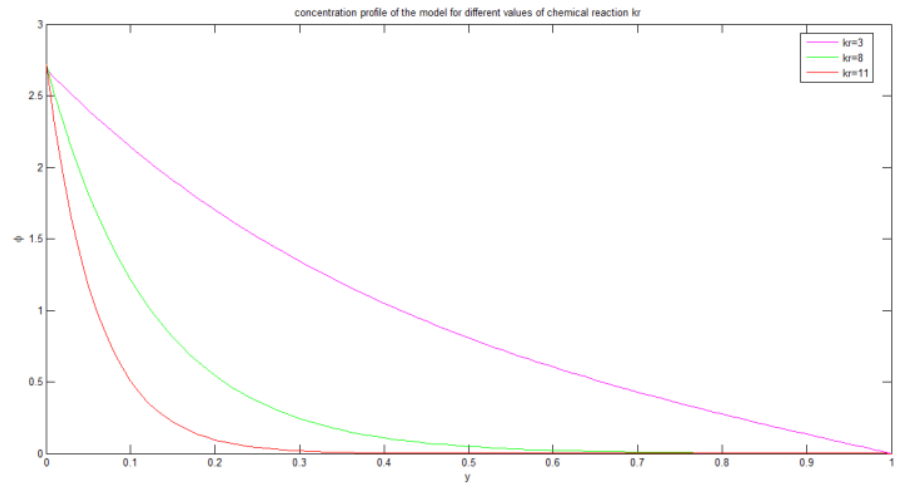

Figure 5 Concentration profile of the model for different values of chemical reaction parameter $\mathrm{Kr}$

Figure 5, shows the influence of chemical reaction parameter $K r$ on concentration profile of the fluid while holding the other parameters constant. The graph of the concentration value is plotted by taking the distance from moving plate to stationary plate denoted by $y$ along the horizontal axis while taking the value of fluid concentration denoted by $\phi$ along vertical axis

The result shows that for a fixed value of chemical reaction parameter $K r$, the value of fluid concentration denoted by $\phi$ starts from a maximum constant value at the moving plate, and as $y$ increases the concentration $\phi$ decreases and reaches zero constant value at the stationary plate. Nevertheless, the measure of fluid concentration at the moving plate is always at a higher value than that at the stationary plate.

As the concentration of fluid at the moving plate is higher than that at the stationary plate, the concentration is expected to move from moving plate towards stationary plate. Fluids diffuse from the locations of higher concentrations to that of lower concentrations. This fact has been supported in this simulation.

Also it can be observed from that simulation: (i) the value of concentration of fluid does not change with the change in $K r$ at the moving plate, (ii) the value of concentration of fluid, occurring between the two plates, decreases with the increase of $K r$ i.e. the value of concentration of fluid is inversely proportional to that of $K r$, and (iii) the value of concentration of fluid goes to zero at the stationary plate irrespective of the value of $K r$.

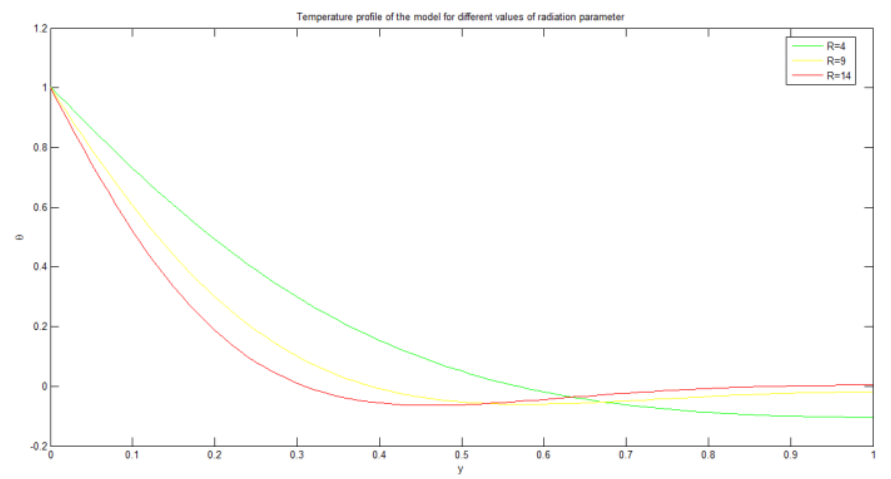

Figure 6 Temperature profile of the model for different values of Radiative parameter $R$

Figure 6, shows the influence of thermal radiation parameter $R$ on transfer of the temperature while holding the other parameters constant. The graph of the temperature is plotted by taking the distance from 
moving plate to stationary plate denoted by $y$ along the horizontal axis while taking the value of temperature denoted by $\theta$ along vertical axis.

The result shows that for a fixed value of thermal radiation parameter $R$, the temperature $\theta$ starts from a constant value at the moving plate. Also as $y$ increases the temperature decreases till it reaches a minimum value. However, thereafter the temperature increases with the increase of $y$ and ultimately the temperature reaches a constant value. Nevertheless, the measure of temperature at the moving plate is always at a higher value than that at the stationary plate.

As the temperature of the moving plate is higher than that of the stationary plate, the temperature is expected to transfer from moving plate towards stationary plate. This fact has been supported in this simulation.

Also it can be observed from that simulation: (i) the value of temperature does not change with the change in $R$ at the moving plate, (ii) the minimum value of temperature, occurs between the two plates, decreases with the increase of $R$ i.e. minimum value of temperature is inversely proportional to that of $R$, and (iii) the value of temperature increases with the increase of $R$ at the stationary plate i.e. the value of temperature is directly proportional to that of $R$.

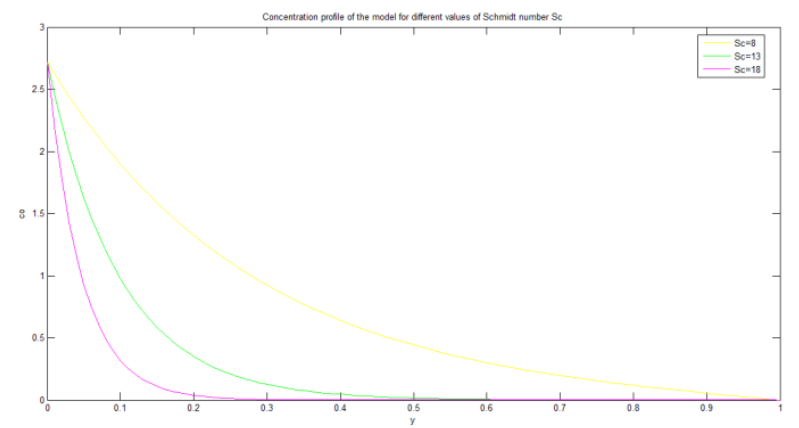

Figure 7 Concentration profile of the model for different values of Schmidt number $S c$

Figure 7, shows the influence of Schmidt Number $S c$ on concentration profile of the fluid while holding the other parameters constants. The graph of the concentration value is plotted by taking the distance from moving plate to stationary plate denoted by $y$ along the horizontal axis while taking the value of fluid concentration denoted by $c o$ along vertical axis.

The result shows that for a fixed value of Schmidt number $S c$, the value of fluid concentration denoted by $c o$ starts from a maximum constant value at the moving plate, and as $y$ increases the concentration $c o$ decreases and reaches zero constant value at the stationary plate. Nevertheless, the measure of fluid concentration at the moving plate is always at a higher value than that at the stationary plate.

As the concentration of fluid at the moving plate is higher than that at the stationary plate, the concentration is expected to move from moving plate towards stationary plate. Fluids diffuse from the locations of higher concentrations to that of lower concentrations. This fact has been supported in this simulation.

Also it can be observed from that simulation: (i) the value of concentration of fluid does not change with the change in $S c$ at the moving plate, (ii) the value of concentration of fluid, occurring between the two plates, decreases with the increase of $S c$ i.e. the value of concentration of fluid is inversely proportional to that of $S c$, and (iii) the value of concentration of fluid goes to zero at the stationary plate irrespective of the value of $S c$.

In general, the concentration decreases with an increase in $S c$. Physically, this is true since the increase in $S c$ implies decrease of molecular diffusivity and hence decreases in concentration boundary layer. 


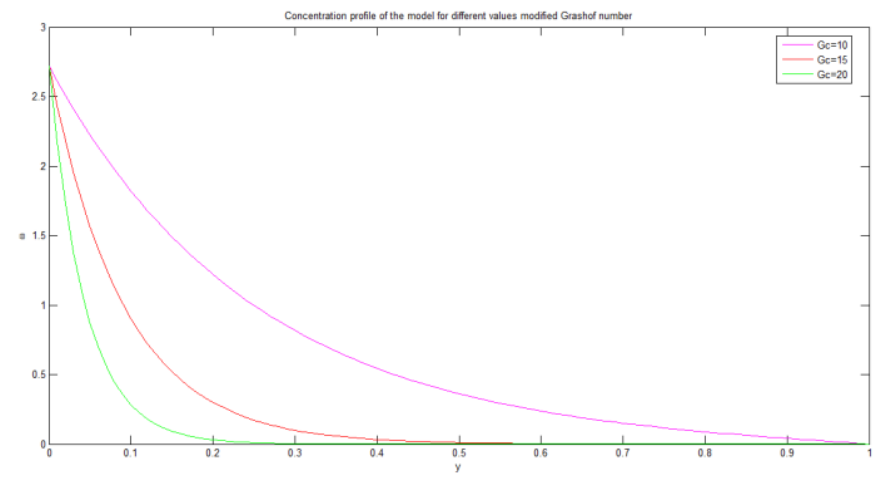

Figure 8 Concentration profile of the model for different values of Modified Grashof number $G c$

In Figure 8, the influence of Modified Grashof number $G c$ on the concentration of the fluid has been studied by simulation. Naturally, the number $G c$ and the concentration are inversely proportional to each other i.e.as Gc increases the velocity of the fluid increases and hence the concentration decreases. The same fact has been even supported by the present simulation study. is given by

We will now study the effects of thermal Grashof and Modified Grashof number on skin friction which

$\bar{\tau}=\mu\left(\frac{d \bar{u}}{d \bar{y}}\right)$ at $y=0$

$\tau=\frac{\bar{\tau} b}{\mu U_{0}}$

Using the non-dimensional quantity

the skin friction equation (39) reduces to

$\tau=\frac{d u}{d y}$ at $y=0$.

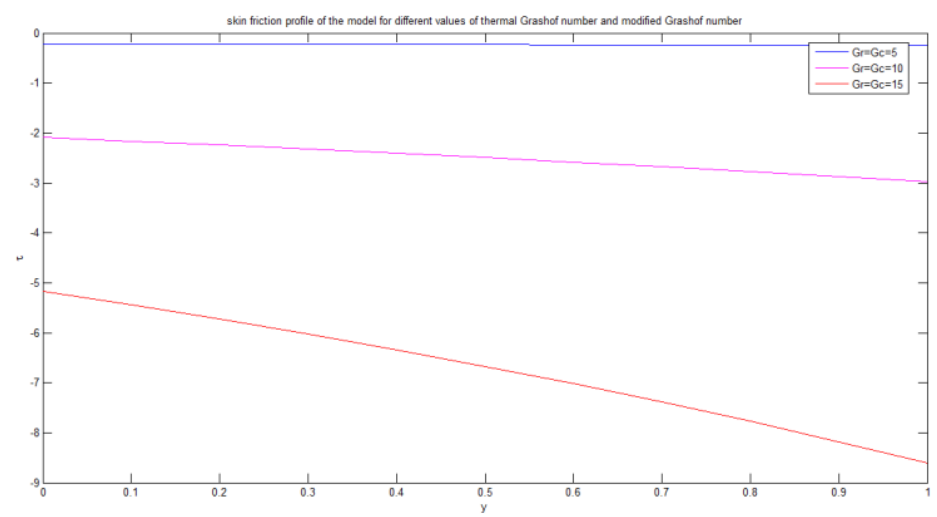

Figure 9 Skin friction profile of the model for different values of thermal Grashof number $\mathrm{Gr}$ and modified Grashof number Gc.

In Figure 9, the influence of skin friction profile of the model for different values of thermal Grashof number and modified Grashof number have been discussed. The graph of the skin friction value is plotted by taking the distance from moving plate to stationary plate denoted by $y$ along the horizontal axis while taking the value of skin friction denoted by $\tau$ along the vertical axis. From the simulation study we have observed that increasing in both thermal Grashof number and modified Grashof number results in decreasing the skin friction. This is due to the presence of permeability of porous medium which has a resistive effect on velocity results in decreasing the velocity of the fluid flow.

\section{Conclusion}

In this paper the influence of thermal radiation and chemical reaction parameter on unsteady Magneto hydrodynamic free convective fluid flow bounded between two infinite vertical Couette plates embedded in a porous medium has been considered.

The configuration of the fluid flow is affected by the vertical motion of the hot plate. Although, the vertical motion of the hot plate is due to the radiation parameter, buoyancy force and shear induced force of the hot plate which helps to move vertical motion in a uniform manner. It is observed the flow pattern of the fluid is affected by radiation parameter, porous medium and induced force. 
Regular perturbation technique is applied to convert the governing non-linear partial differential equations into a system of ordinary differential equations which can be solved analytically.

In addition, the effects of various parameters viz. permeability of porous medium, thermal Grashof number, frequency parameter, radiation parameter, chemical reaction parameter and Schmidt number on velocity, temperature and concentration has been discussed.

\begin{tabular}{|c|c|}
\hline Variables and parameters & Meaning \\
\hline$G r$ & Thermal Grashof number \\
\hline $\mathrm{k}$ & Permeability of porous medium \\
\hline Gc & Modified Grashof number \\
\hline$S c$ & Schmidt number \\
\hline$t$ & Time \\
\hline$M$ & Hartmann number \\
\hline $\operatorname{Pr}$ & Prandtl number \\
\hline$q_{r}$ & Radiative heat flux \\
\hline$k_{r}$ & Chemical reaction parameter \\
\hline$R$ & Radiation parameter \\
\hline$D$ & Mass diffusivity \\
\hline $\bar{T}$ & Temperature of the fluid in the boundary layer \\
\hline $\bar{T}_{m}$ & Temperature of the moving plate \\
\hline$\overline{\bar{T}}_{b}$ & Temperature of the stationary plate \\
\hline$\overline{\bar{U}}$ & Free stream velocity \\
\hline$U$ & Dimensionless free stream velocity \\
\hline $\bar{u}$ & Velocity component in $\bar{x}$ \\
\hline $\bar{v}$ & Velocity component in $\bar{y}$ \\
\hline $\bar{x}, \bar{y}$ & Cartesian coordinates \\
\hline$x, y$ & Dimensionless Cartesian coordinates \\
\hline $\bar{C}_{m}$ & Concentration at plate $(\mathrm{y}=0)$ \\
\hline $\bar{C}_{b}$ & Concentration at plate $(y=b)$ \\
\hline$C$ & Dimensionless concentration \\
\hline$C_{p}$ & Specific heat at constant pressure \\
\hline $\mathrm{J}$ & Electric current density \\
\hline$b_{1}$ & Induced magnetic field \\
\hline$B_{0}$ & Strength of magnetic field \\
\hline$g$ & Acceleration due to gravity \\
\hline Greek letters & Meaning \\
\hline$\beta$ & Thermal expansion coefficient \\
\hline$\beta_{c}$ & Concentration expansion coefficient \\
\hline$\varepsilon$ & Amplitude of free stream velocity \\
\hline$\omega$ & Frequency of oscillation \\
\hline$\theta$ & Dimensionless temperature \\
\hline$\kappa$ & Thermal conductivity \\
\hline$v$ & Kinematic viscosity \\
\hline$\mu$ & Dynamic viscosity \\
\hline$\alpha$ & Thermal diffusivity \\
\hline$\sigma$ & Electric conductivity \\
\hline
\end{tabular}

\section{References}

[1]. Nield, D. A. and Bejan, A. 1998: Convection in porous media. 2nd edition, Springer Verlag, Berlin.

[2]. Hossain, M. A. and Begum, R. A.:1985: The effects of mass transfer on the unsteady free convection flow past an accelerated vertical porous plate with variable suction, Astrophysics.

[3]. A. Raptis 1983: Unsteady free convection flow through a porous medium, Int. J. Engin. Sci. 21, 345-348.

[4]. A. Raptis and C. P. Peridikis 1985: Oscillatory flow through a porous medium by the presence of free convective flow, Int. J. Engin. Sci. $23,51-55$.

[5]. A. K. Singh 2002: MHD free convective flow through a porous medium between two vertical parallel plates, Ind. J. Pure and Appl. Phys. 40, 709-713.

[6]. Sharma, P. K. 2004: Unsteady effect on MHD free convective and mass transfer flow through porous medium with constant suction and constant heat flux in rotating system. Acta Ciencia Indica Mathematics, 30(4): 873-880.

[7]. Helmy, K.A. 1998: MHD Unsteady Free Convective Flow past a Vertical Porous Plate. Journal of Applied Mathematics and Mechanics, 78, 255-270.

[8]. Hossain, Md. Anwar and Pop, I. (2001): Radiation Effects on Free Convection over a Vertical Flat Plate Embedded in a Porous Medium with High Porosity. International Journal of Thermal Sciences, 40, 289-295.

[9]. Ibrahim, S.Y. and Makinde, O.D. 2011: Radiation Effect on Chemically Reacting MHD Boundary Layer Flow of Heat and Mass Transfer through a Porous Vertical Flat Plate. International Journal of Physical Sciences, 6, 1508-1516.

[10]. Das, S., Jana, M. and Jana, R.N. 2011: The effect of radiation on Natural Convection near a Vertical Plate Embedded in Porous Medium with Ramped Wall Temperature. Open Journal of Fluid Dynamics, 1, 1-11. 
[11]. Manna, S.S., Das, S. and Jana, R.N. 2012: Effects of Radiation on Unsteady MHD Free Convective Flow Past an Oscillating Vertical Porous Plate Embedded in a Porous Medium with Oscillatory Heat Flux. Advance in Applied Science Research, 3, 37223736 .

[12]. Ibrahim, Md. S. 2013: Radiation Effects on Mass Transfer Flow through a Highly Porous Medium with Heat Generation and Chemical Reaction. Hindawi Publishing Corporation ISRN Computational Mathematics, Article ID: 765408.

[13]. Anjalidevi, S.P. and R. Kandasamy 2000: Effects of a chemical reaction heat and mass transfer on MHD flow past a semi-infinite plate. Z. Angew. Math. Mech., 80:697-701.

[14]. E. M. A. Elbashbeshy, D. M. Yassmin and A. A. Dalia 2010: Heat Transfer Over an Unsteady Porous Stretching Surface Embedded in a Porous Medium with Variable Heat Flux in the Presence of Heat Source or Sink, African Journal of Mathematics and Computer Science Research Vol. 3, No.5, Pp. 68-73,

[15]. Chin, K. E., Nazar, R., Arifin, N. M., and Pop, I. 2007: Effect of variable viscosity on mixed convection boundary layer flow over a vertical surface embedded in a porous medium. International Communications in Heat and Mass Transfer, 34(4): 464-473

[16]. Angirasa, D., Peterson, G. P. and Pop, I. 1997: Combined heat and mass transfer by natural convection with opposing buoyancy effects in a fluid saturated porous medium, Int. J. Heat Mass Trans., 40(12), 2755- 2773.

[17]. Yih, K. A. 1997: The effect of transpiration on coupled heat and mass transfer in mixed convection over a vertical plate embedded in a saturated porous medium. International Communications in Heat and Mass Transfer, 24(2): 265-275.

[18]. Anand Rao, J. and Shivaiah, S. 2011: Chemical reaction effects on an unsteady MHD Free convection flow past an infinite vertical porous plate with constant suction, Chem. Ind. Chem. Eng. Q., 17: 249-257.

[19]. Anand Rao, J. and Shivaiah, S. 2011: Chemical reaction effects on an unsteady MHD flow past a semi-infinite vertical porous plate with viscous dissipation. Journal of Applied

[20]. Mathematics and Mechanics (English Edition), 32, 8: 1065-1078.

[21]. Samad M.A. and Rahman M.M. 2006: Thermal radiation interaction with unsteady MHD flow past a vertical porous plate immersed in a porous medium. Journal of Naval Architecture and Marine Engineering, Vol.3, Pp.7-14.

[22]. Brewster M.A (1992): Thermal Radiative Transfer and Properties. - New York: John Wiley and Sons.

[23]. Pawan Kumar Sharma, Mukesh Dutt (2014.): MHD oscillatory free convection flow past parallel plates with periodic temperature and concentration, Universal Journal of Applied Mathematics, Vol. 2, No. 7, Pp 264 - 75, Doi: 10.13189/ujam.2014,020702. 